\title{
lieuxdits \#4
}

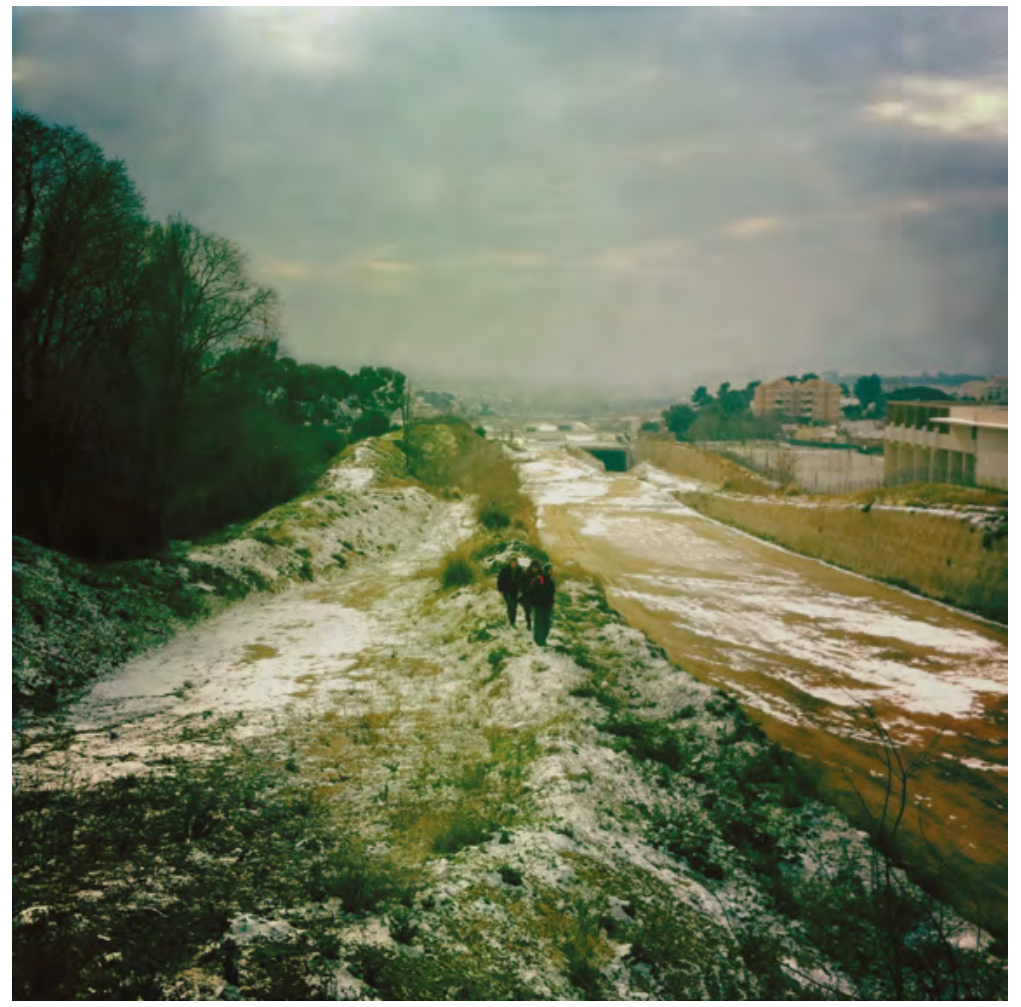

Référence bibliographique :

Renaud Pleitinx, "Le Champ des Possibles", lieuxdits\#4, décembre 2012, pp.17-22.

\section{La revue lieuxdits}

Faculté d'architecture, d'ingénierie architecturale, d'urbanisme (LOCI)

Univesrité catholique de Louvain (UCL).

Éditeur responsable : Jean-Paul Verleyen, place des Sciences, 1 - 1348 Louvain-la-Neuve

Comité de rédaction : Martin Buysse, Damien Claeys, Gauthier Coton,

Jean-Philippe De Visscher, Guillaume Vanneste, Jean-Paul Verleyen

Conception graphique : Nicolas Lorent

Impression : école d'imprimerie Saint-Luc Tournai

\section{(C) (i) (8) $\Theta$}




\section{Le Champ des possibles}

Contribution spéculative à la Réflexion sur la Recherche par le projet

\section{Renaud Pleitinx}
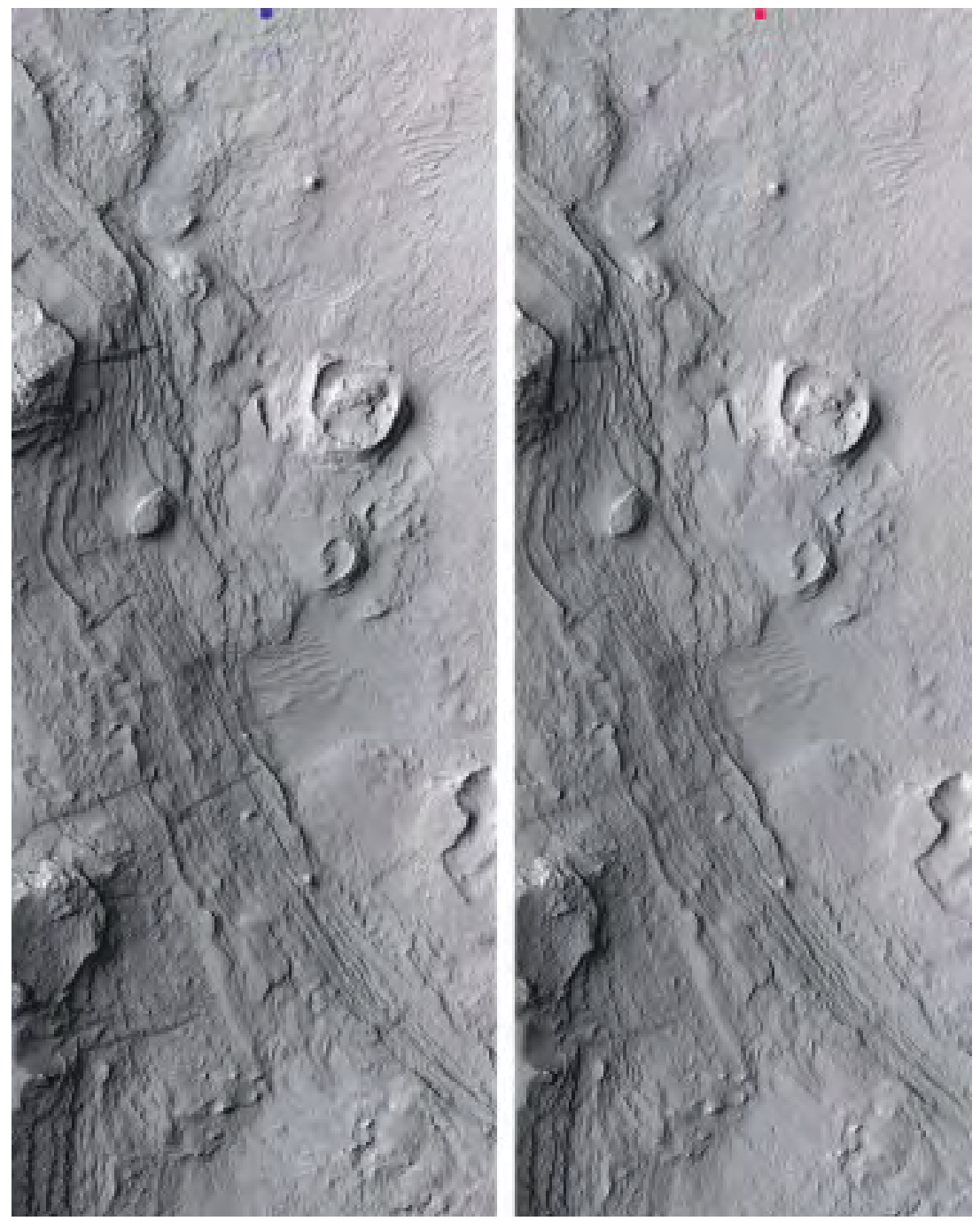

Depuis peu, une discipline réclame le droit d'exister à l'Université et plus particulièrement dans les facultés d'architecture. Elle porte un nom déjà, en forme de programme : Recherche par le projet d'architecture (RPP). Mais, ses statuts autant que ses devoirs sont encore trop mal définis pour lui permettre d'accéder aussitôt au titre de recherche universitaire. II lui faut encore montrer patte blanche, convaincre que sa pratique participe bien des missions de l'Université.

Le propos à suivre est une contribution à la construction des arguments en faveur de la RPP ; il est donc de parti pris. Pourtant, il tend à problématiser la RPP, en réfutant les sophismes complaisants mais surtout en lui opposant de sévères objections. L'enjeu de cette approche sceptique est ultimement de retourner les arguments à charge, comme on le fait d'un gant, pour faire valoir le domaine spéculatif propre à la RPP et en déduire les démarches qui garantissent finalement son "objectivité". 


\section{Deux traditions à concilier}

Pour permettre de poser les questions que soulève la RPP, il est nécessaire de dissiper une confusion courante sur laquelle s'appuie un raisonnement controuvé, qui, pour abonder dans le sens de la RPP, fournit de manière dommageable une raison de ne pas en interroger les fondements, de ne jamais la mettre en cause.

En accordant au vocable son sens usuel, on peut affirmer que les architectes "cherchent" lorsqu'ils élaborent un ouvrage, lorsqu'ils s'adonnent au projet d'architecture. Tirant argument d'une imprécision, d'aucuns en concluent que les architectes sont des "chercheurs" et conjointement que la pratique du projet d'architecture s'inscrit de fait dans les missions de "recherche" confiées à I'Université. L'argument, qui n'est pas dénué de malice, repose tout entier sur un usage peu scrupuleux du vocable "recherche". Le procédé est connu, en désignant deux choses différentes par un même mot on produit le mythe de leur profonde identité. On induit ainsi une confusion dont profite la démonstration : l'architecte cherche, donc le projet d'architecture est une recherche, et par la même occasion l'architecte est un chercheur. L'usurpation de titre n'est pas la pire conséquence de cette confusion. II faut surtout s'inquiéter du fait qu'elle annule logiquement toute question. Si le projet d'architecture est par la seule force des mots assimilé à une recherche universitaire, poser la question d'une RPP devient trivial.

Pour néanmoins laisser une chance à la question, il faut admettre que la recherche universitaire et le projet d'architecture relèvent de traditions disciplinaires distinctes dont les tâches et les centres d'intérêts diffèrent radicalement. Refuser de les confondre est une condition à remplir pour pouvoir énoncer les questions que pose leur rencontre inopinée : comment le projet d'architecture, tel que pratiqué par les architectes, peut-il s'inscrire dans la recherche confiée aux chercheurs universitaires ? Quels accords sont nécessaires entre ces deux traditions pour donner sens et vigueur à l'expression "Recherche par le projet" ?
Il importe de rappeler, à toutes fins utiles, ce qui distingue la recherche universitaire et le projet d'architecture. Depuis la fondation des universités, il y a bientôt mille ans, la recherche universitaire est consacrée, quels qu'en soient les protocoles autorisés, à l'établissement $d u$ savoir, c'est-à-dire, au bas mot, à la saisie conceptuelle d'objets, à leur catégorisation et à leur compréhension. Cette tâche, mobilise en première instance notre faculté langagière appliquée à nos capacités perceptives et symboliques. Autrement dit, l'élément dans lequel baigne le chercheur universitaire - celui que l'on nomme aussi : le savant - est essentiellement verbal. En témoignent de manière tangible les précipités de paroles que sont : les mémoires, les articles scientifiques et les thèses dont regorgent les bibliothèques facultaires.

Le projet d'architecture en revanche est dévolu à l'élaboration de l'habitat qui précède sa mise en œuvre et en service. Cette élaboration mobilise autant notre faculté à produire, à formuler, à mettre au point un artefact, qu'à légitimer nos désirs. II s'agit là de deux compétences distinctes qui pour être auss rationnelles que le langage n'en sont pas moins indépendantes. Ainsi, l'architecte, à qui la division des métiers a confié le soin du projet d'architecture, fait à la fois preuve d'une agilité artistique, proprement technico-productive, et en même temps traverse peu ou prou les affres d'un débat éthico-moral.

Par vocation donc, le savant et l'architecte opèrent au sein de dimensions distinctes de la culture. Cela veut dire que les points d'application de leurs efforts respectifs siègent en des plans séparés et que leurs résultats - connaissance d'une part et habitat projeté de l'autre - ont des raisons radicalement différentes. On comprend alors que la recherche savante n'est pas en mesure de produire un habitat, pas même un projet d'habitat, mais aussi que le projet d'architecture n'est pas en soi producteur de savoir.

Si l'Université a pour mission d'établir des savoirs, il est requis que la RPP - puisqu'elle souhaite trouver place à I'Université - ait pour résultat un surcroît de connaissance. Or sur ce point, le projet d'architecture fait définitive- 
ment défaut. La question se pose alors : comment admettre qu'il puisse compter parmi les démarches de la recherche universitaire?

D'aucuns ont proposé une parade au mutisme de l'habitat et à la foncière incompétence encyclopédique du projet d'architecture. Elle consiste à envisager le projet d'architecture, non comme une fin en soi, mais comme "un instrument de connaissance".

II est remarquable qu'une option semblable ait été défendue dans le milieu littéraire. De nombreux auteurs ont en effet défini le roman comme un "instrument de connaissance". Les tenants de cette ligne accordent un puissant pouvoir d'élucidation à la fiction littéraire, pouvoir capable de rivaliser avec l'efficacité des démarches scientifiques.

S'agissant d'architecture, accorder au projet le statut d'instrument, c'est en faire un auxiliaire de la connaissance. C'est admettre, en d'autres termes, que l'étude savante et le projet d'architecture puissent collaborer de telle sorte que la première constitue un savoir par l'entremise des résultats du second, comme l'astronomie s'appuie sur l'observation d'images saisies par des télescopes. Considérer le projet d'architecture comme un moyen de connaissance donne un vigoureux espoir à la RPP. Se munissant du projet d'architecture comme d'un instrument et en accordant une place nécessaire à l'acquisition de connaissance, elle serait en effet apte à remplir l'exigence minimale requise par les statuts de I'Université : constituer du savoir.

Cependant, la métaphore de l'instrument d'optique, malgré son élégance, présente pour le moins deux difficultés. Deux questions subsistent en effet qui mettent en cause le supposé pouvoir d'élucidation du projet d'architecture. La première question interroge le statut des objets que le projet porte à notre connaissance et s'inquiète du champ d'étude qui serait celui de la RPP. Si à titre d'hypothèse on peut admettre que le projet est un instrument de connaissance, peut-on préciser à quelles choses, à quel réel, il donne finalement accès ? De prime abord, le résultat patent d'un projet d'architecture n'est rien de plus qu'un habitat projeté dont l'existence est conditionnelle. Mais pour la connaissance, quelle sorte d'objet est-ce là qui n'existe pas sinon sur un mode potentiel ? D'aucun répondrait à bon droit : une chimère. Et au regard des recherches attelées à la compréhension des réalités, quelle consistance peut avoir le champ d'investigation ainsi offert à la recherche par le biais du projet sinon celle, toute onirique, du pays des merveilles?

La seconde question qui met en doute la fiabilité du projet d'architecture comme instrument de connaissance porte sur l'engagement erratique qui fonde tout projet. Quelle peut être "l'objectivité" d'une RPP dont le point de départ est toujours une proposition, un parti, autant dire plus simplement : un pari ? Si tout projet porte au jour les conséquences d'une supposition, comment accepter que s'immisce dans la recherche une telle incertitude ? Incertitude elle-même suspendue à la personnalité de l'auteur du projet, aux usages qui sont les siens en matière d'habitat, à ses obsessions et ses travers, à ses faiblesses ou à son habileté.

La délimitation d'un champ de connaissance consistant ainsi que l'objectivité ou l'impartialité de la connaissance sont des exigences scientifiques incontournables. On comprend alors que ces deux questions portent un sérieux coup à l'ambition de faire admettre la RPP au sein de l'Université, quand bien même le projet serait considéré comme un instrument de connaissance.

Pour un savoir du possible

Si on souhaite ne pas laisser choir la RPP dans une impasse épistémologique, il convient de lever les doutes légitimes auxquels elle se trouve exposée. Pour ce faire, à vrai dire, il suffit de confirmer leurs arguments. Ce n'est en effet qu'en admettant le caractère radicalement chimérique des résultats du projet d'architecture que l'on pourra "cerner" le domaine propre de la RPP et plus loin définir les protocoles par lesquels elle est en mesure d'accéder à l'objectivité. Ainsi, nous ferons des apparentes faiblesses de la RPP ses meilleurs atouts.
1 - Musil R., L'homme sans qualités, p. 40

2-Hallward P., Toutestpossible dans La Revue internationale des Livres et des Idées. 
Abruptement, on pourrait proposer de fonder la RPP sur deux affirmations incontestables :

1. Un projet (d'architecture) est un coup de sonde dans le champ infini des (habitats) possibles. Autrement dit, le résultat de tout projet d'architecture, un habitat projeté, est un échantillon des possibilités de l'habitat.

2. Le domaine d'étude offert à la RPP d'architecture est le champ des habitats possibles.

On déduirait aisément de ces deux propositions que la tâche de la RPP consiste à étudier les possibilités offertes à l'habitat en général, voire à un habitat particulier. Connaître, c'est-à-dire constater et expliquer, l'espace des possibles, telle serait en définitive la tâche de la RPP.

II est remarquable que le champ des possibles inclut de fait la réalité. Car celle-ci n'est jamais qu'un possible parmi d'autres. Cela implique qu'une connaissance du possible ne peut faire exception de ce qui existe. De ce fait, la RPP ne pourra ignorer la diversité des habitats existants. Mais elle ne pourra pas s'en contenter. On peut en effet s'attendre à ce que le champ des possibles possède une extension infinie. Chaque secteur de l'habitat (territoire, ville, logement, équipement, ameublement, véhicule), chaque situation (qu'elle soit particulière ou générale) recèlent en effet une quantité inépuisable de potentialités. Pour autant, le champ des possibles pourrait présenter des aires de densités variables, comporter des limites internes. Des forces pourraient y imprimer des mouvements qui configureraient un territoire accidenté, déchiré, plissé. II devrait ainsi exister une géographie du possible dont la RPP aurait à rendre compte.

Constituer un savoir du possible réclame sans doute une épistémologie adéquate qui ne restreigne pas la portée du domaine de la connaissance à l'existant mais l'étende au champ du possible. Pour qu'un tel mode de pensée puisse s'épanouir, il faut sans doute une sensibilité propice à son émergence ; "un sens du possible", comme le définit
Robert Musil : "la faculté de penser tout ce qui pourrait être "aussi bien", et de ne pas accorder plus d'importance à ce qui est qu'à ce qui n'est pas'." Entraînés à imaginer ce que les choses pourraientêtre, par de-delà ce qu'elles sont, les architectes ont un sens du possible affuté, qualité que la RPP réclame.

S'agissant par ailleurs d'une pensée du possible, il faut noter l'émergence récente d'un courant philosophique dénommé : le réalisme spéculatif (RS) dont les arguments pourraient aider à stabiliser les fondements épistémologiques de la RPP. Si la majeure partie de ses protagonistes sont d'origine anglo-saxonne, la figure de proue du RS est hexagonale : il s'agit de Quentin Meillassoux. Le nœud du propos de Meillassoux est la démonstration rationnelle de l'absolue contingence des lois naturelles, et finalement de toutes choses. Les thèses radicales de Quentin Meillassoux ré-ouvrent le champ de l'ontologie. Comme l'affirme Peter Hallward : "Pour Meillassoux, tout comme pour Platon ou Hegel, la préoccupation première de la philosophie concerne la nature de la réalité absolue, mais telle que Meillassoux la conçoit, la nature de cette réalité requiert que la philosophie s'astreigne à penser non "ce qui est, mais ce qui peut possiblement être". (...) Si Meillassoux peut être défini comme un "réaliste", alors la réalité qui le préoccupe n'implique pas tant les choses telles qu'elles sont, que la possibilité qu'elles puissent toujours être autrement ${ }^{2} . "$

Sens du possible et pensée spéculative seraient opportunément mis au service la RPP. Ils aideraient du moins à prendre la mesure de la tâche et à ne pas trembler face à son énormité. Car, le possible n'est pas seulement le probable, il renferme aussi bien l'improbable. A priori, rien ne devrait obliger la RPP à se plier aux urgences du métier d'architecte sur lequel pèse l'incontournable exigence de faisabilité prochaine. Toutes les contrées du possible devraient être investiguées. On ne négligerait ainsi aucune fantaisie, aucune utopie. Alice courant derrière le lapin blanc deviendrait l'exemple à suivre ; le docteur Faustroll serait un maître ; la science-fiction un paradigme. Comme toujours lorsque le savoir gagne en autonomie, il faudra répondre à la 
question pragmatique : quelle utilité peut avoir une RPP ? Vouée à la connaissance du possible, la RPP n'aurait pas pour vocation de prévoir ou prédire l'avenir en spéculant sur un enchaînement de causes toujours douteux dès lors qu'il s'agit de l'Histoire des hommes. Elle devrait encore moins prétendre imposer un avenir. S'il ne faut attendre de la RPP aucune prédiction et s'il faut lui refuser toute autorité prescriptive, il n'est cependant pas exclu que l'une ou l'autre des potentialités mises à jour et étudiées par celle-ci, puisse être historiquement appropriée - être choisie par des gens - et une fois mise en œuvre, devenir réalité. On verrait alors l'intérêt pratique de la RPP : faire saillir et méditer des points de décision collective quant à notre habitat.

En résumé, pour peu qu'elle ne se suffise pas du projet mais envisage ce dernier comme un instrument de connaissance, la RPP pourrait prétendre à un domaine de compétence propre : les possibilités de I'habitat, champ d'investigation qui inclut de fait la réalité, mais la déborde de très loin. Rétroactivement, l'incertitude foncière du projet d'architecture s'avère être la condition de possibilité d'une investigation du possible et par la même occasion la pierre angulaire de la RPP. Car c'est très précisément l'engagement conditionnel de tout projet, le pari inhérent à son parti, qui force les limites de ce qui est, et conduit, en un saut sans mesure, au domaine du possible. Si un projet ouvre comme une porte sur le possible, son incertitude primitive en est la clef. Finalement, si on admet que la RPP a pour objet les possibilités de l'habitat, le projet d'architecture s'avère être son instrument indispensable.

\section{Quelques points de méthode}

De sa définition même et de la nature de son champ d'étude, on peut déduire les démarches de la RPP. Par définition, la RPP devrait inclure deux types de démarche : projective d'une part, et cognitive d'autre part. Comme "instruments" de connaissance, les démarches inhérentes au projet (décision, élaboration par le dessin) seraient nécessairement premières. Les démarches cognitives supposant un habitat projeté, seraient donc consécutives au projet. Cet ordre n'exclurait pas d'éventuelles itérations. On admettrait bien sûr qu'un territoire déjà balisé fasse entrevoir des régions encore inexplorées qui justifient de nouvelles investigations.

Dans un premier temps donc, la tâche assignée au projet serait de faire émerger des possibles ; son objectif serait d'échantillonner les possibilités. De prime abord tout projet, dans la mesure où il grossit l'échantillon, serait bon à prendre. Une complète liberté d'action devrait être laissée aux architectes quant à leurs moyens d'investigations. Peu importe que le projet prenne appui sur les formes de l'habitat ou sur les paramètres d'une situation. Peu importe le style adopté ou l'éthique gouvernant les projets. Peu importe surtout la probabilité attachée aux possibilités mises à jour. La seule contrainte véritable serait de multiplier les projets. Pour permettre de baliser correctement le champ des possibilités étudié, il conviendrait en effet de répéter les coups de sonde. Ceci obligerait les auteurs de projet impliqués dans la recherche à diversifier leurs points de départ, leurs angles d'approches, pour varier leurs propositions. La multiplication des projets demanderait à leurs auteurs de suspendre leurs déterminations personnelles pour dépasser leurs préjugés, leurs attendus, pour aller là où ils ne sont pas et même là où ils se refuseraient d'aller. La tâche serait opportunément remplie de manière collective, mais si un projeteur devait se retrouver seul, il lui faudrait composer, tel Fernando Pessoa sous une foule d'hétéronymes. Le projet d'architecture mis au service de la connaissance du possible serait ainsi une démarche impersonnelle ; rendue méthodiquement indifférente à toute signature. La personnalité des auteurs n'aurait ainsi pas d'incidence sur l'échantillonnage du possible, et partant la RPP pourrait prétendre à l'impartialité voire à l'objectivité.

Consécutif à ce premier temps exploratoire, le moment réflexif de la recherche aurait pour tâche d'étudier les habitats projetés. Muni d'un échantillon de possibilités, on pourrait en première 
approche décrire la structure du possible. En comparant tant la mise au point technique des habitats projetés que leurs partis constitutifs, on relèverait les différences qui les opposent autant que les similitudes qui les apparentent. En outre, envisageant les habitats projetés comme des unités combinables, on pourrait tester leur compatibilité ; voir s'ils peuvent s'emboîter les uns dans les autres ou s'enchaîner les uns aux autres. On irait plus loin encore, en étudiant les transformations admises par les divers habitats projetés. Les questions seraient alors : considérant deux habitats possibles quelles opérations permettent de passer de l'un à l'autre? Que faut-il retrancher, ajouter ou faire varier? Que faut-il par ailleurs admettre ou refuser sinon apprendre à apprécier? La prise en compte de ces transformations ouvrirait en définitive la question délicate des conditions de faisabilité des projets. Elle mettrait en évidence les obstacles ou les dogmes qui empêchent ou interdisent la transformation de tel habitat en tel autre. Point à point, on pourrait ainsi tracer une carte du possible. On y verra apparaître les contours diffus du domaine de la réalité, car la plupart des projets se rassembleront probablement aux marges de ce qui existe déjà. Mais on y verra aussi des points esseulés aux confins du possible, fruits de quelques architectes aventureux qui auraient perdu ou refusé d'exercer leur sens des réalités. Dans la densité moyenne des possibles peu à peu révélés, apparaîtront sûrement des amas concentriques indiquant l'existence d'options en apparence incontournables : des dispositifs indispensables ou des valeurs inamovibles sur lesquelles nombres de projets auront insisté. II faudra relever scrupuleusement ces lieux de convergence. Ils permettraient de fonder quelques certitudes quant aux tenants et aboutissants de I'habitat. Et puis, sur le tableau pointilliste des localités inventoriées, apparaîtront peut-être des régions oubliées, des territoires tabou où aucun projet ne semble ni pouvoir ni vouloir s'établir. II faudra prêter grande attention à ces friches du possible ; savoir ce qui en barre l'accès ; trouver les moyens de le forcer. Car dans ces contrées dédaignées réside peutêtre l'invention véritable.
La rencontre inopinée de la recherche universitaire et du projet d'architecture a fait surgir l'idée d'une RPP. II s'est agi de montrer ici qu'aucun ne perdrait au change. Chacun devrait certes renoncer à ses habitudes ; du côté de l'Université, il faudrait ne plus restreindre la recherche au domaine des réalités ; du côté du projet d'architecture, il faudrait renoncer à la signature singulière et au droit d'auteur. Mais, un domaine d'étude illimité serait ouvert - une mine à exploiter - dont l'Université ne pourrait que se féliciter, et le projet d'architecture serait promu au rang de démarche scientifique indispensable à l'étude d'un champ, celui des possibles.

\section{Bibliographie}

HALLWARD, Peter, "Tout est possible", dans La Revue internationale des Livres et des Idées,2009.

MASSON, Olivier et LEDENT, Gérald, Le projet d'architecture à l'épreuve de la recherche, un profit pour l'enseignement, texte non publié.

MUSIL, Robert, L'homme sans qualités, Traduit de l'allemand par Philippe Jaccottet, Seuil, 2004, coll. "Points". 